\title{
Face Representation and Perceptual Processing in Mask-making
}

\author{
Nina Prescott \\ Department of Population Health Science \& Policy, Icahn School of Medicine at Mount Sinai, \\ New York NY 10029 USA; nina.prescott@mssm.edu
}

\section{Daniel Graham}

Department of Psychology, Hobart \& William Smith Colleges, Geneva NY 14456 USA; graham@hws.edu

Correspondence: graham@hws.edu; Tel.: +1-315-781-4526

\begin{abstract}
Mask-making is widely distributed in human culture and shows remarkable diversity. How are faces represented in masks, and how are these representations processed in the human brain? Here we asked if masks show systematic differences in structure and processing compared to natural faces. First, we assembled a large, diverse database of 138 standardized mask images and measured structural features and ratios. We find that masks show an increased length ratio exaggeration compared to painted portraits and natural faces, as well as larger variance in the length ratio compared to portraits and natural faces. Then, in order to understand how mask representations of the human face are processed in the brain, we used a rapid symmetry detection task to compare upright and inverted stimuli. We predicted that there would be an inversion effect, namely that symmetry detection accuracy would be higher for upright compared to inverted stimuli, indicating the involvement of high-level face-specific processing. Instead, while performance for upright and inverted masks was significantly above chance, there was no significant difference across subjects in performance for upright versus inverted masks $(\mathrm{N}=40)$. This result is consistent with the notion that masks do not fully engage high-level cortical processing systems specialized for the human face. Together, these studies suggest that masks are designed and processed in a way that may be distinct from how natural faces are processed. We conclude that handmade masks can serve purposes beyond face representation, despite masks' superficial functionality as face surrogates.
\end{abstract}

Keywords: perception; face processing; symmetry; pareidolia; face inversion

Acknowledgments: We thank Margaret Coldiron (University of Essex) for her many helpful comments during this project. We received support for this work from a HWS Faculty Research Grant to D.G. 


\title{
Face Representation and Perceptual Processing in Mask-making
}

\author{
Nina Prescott ${ }^{1}$ and Daniel Graham ${ }^{2 *}$ \\ 1 Department of Population Health Science \& Policy, Icahn School of Medicine at Mount \\ Sinai, New York NY 10029 USA; nina.prescott@mssm.edu
}

2 Department of Psychology, Hobart \& William Smith Colleges, Geneva NY 14456 USA; graham@hws.edu

* Correspondence: graham@hws.edu; Tel.: +1-315-781-4526

\begin{abstract}
Mask-making is widely distributed in human culture and shows remarkable diversity. How are faces represented in masks, and how are these representations processed in the human brain? Here we asked if masks show systematic differences in structure and processing compared to natural faces. First, we assembled a large, diverse database of 138 standardized mask images and measured structural features and ratios. We find that masks show an increased length ratio exaggeration compared to painted portraits and natural faces, as well as larger variance in the length ratio compared to portraits and natural faces. Then, in order to understand how mask representations of the human face are processed in the brain, we used a rapid symmetry detection task to compare upright and inverted stimuli. We predicted that there would be an inversion effect, namely that symmetry detection accuracy would be higher for upright compared to inverted stimuli, indicating the involvement of high-level face-specific processing. Instead, while performance for upright and inverted masks was significantly above chance, there was no significant difference across subjects in performance for upright versus inverted masks $(\mathrm{N}=40)$. This result is consistent with the notion that masks do not fully engage high-level cortical processing systems specialized for the human face. Together, these studies suggest that masks are designed and processed in a way that may be distinct from how natural faces are processed. We conclude that handmade masks can serve purposes beyond face representation, despite masks' superficial functionality as face surrogates.
\end{abstract}

Keywords: perception; face processing; symmetry; pareidolia; face inversion

\section{Introduction}

Mask making is a "ubiquitous" cultural practice (Young-Laughlin \& Laughlin, 1988). Three-dimensional representations of the face are among the first evidence of symbolic expression in human ancestors (Bednarik, 2006). As an art form, masks are particularly useful because they use the unchanging human face as a template. In practice, however, masks often represent a belief or idea in physical form (Mack, 1994), in varied ways: vibrant colors, an assortment of patterns and materials, and diverse facial expressions, to name a few (see Figure 1). With such variation, defining masks as a class proves challenging (Edson, 2009). We define masks broadly as a 3-D representation of a face-like structure, typically worn over the face, in order to expansively observe this face representation and compare it to others. 
However, despite the natural relevance of world mask-making traditions to face perception and to empirical aesthetics, systematic research of visual features and processing in human viewers is minimal. ${ }^{1}$ Aronoff et al. (1988) found that angularity in non-Western masks portrays a sense of threat to Western viewers. This study also suggests that face features such as upturned eyebrows are not reliable cues in this respect. For Japanese Noh masks, Lyons et al. (2000) observed that the viewing angle of the mask's features may indicate the intended expression: they found evidence that viewing a Noh mask from a forward-tilted perspective caused features to curve upwards, portraying happiness. Kawai et al. (2014) extended this work, altering shadows cast on Noh masks to identify varied emotional recognition.

Any sample of masks from world cultures will also show variety in purpose-some may be used for theatrical or dance performances, like the masks used in ancient Japanese dance, while others may be created for funerary purposes, such as ancient Egyptian and Roman mummy masks (Mack, 1994). Given this diversity in form and function, we are interested in investigating what is typical in mask structure, especially given evidence that basic determinants of face preference appear universal in humans (Bruce \& Young, 2012; Calder, Rhodes, Johnson, \& Haxby, 2011; Germine et al., 2015).

The normative aspects of mask structure are also interesting in relation to handmade representations of the face in other media. Previous studies of representations of the face in painted portraiture suggest that such representations do not adhere to aesthetic norms for the structure of the natural female face (Graham et al., 2014). In particular, there is evidence that frontal female portraits have higher length ratios (i.e., ratio of eye-mouth distance to face length; see Figure 2 for example facial measurements) than natural faces; since average face structure is typically preferred, this result also means that portraitists are not systematically altering models' face structure to be more average and therefore more attractive. Interestingly, while composite portraits do show increased attractiveness with a greater degree of averaging (as with faces), there also appears to be a shifted aesthetic norm for individual portraits towards higher length ratios in images with digitally manipulated structure (Graham et al., 2014). The first phase of the present study, Experiment 1, investigates typical basic face structure and analyzes differences in length ratios within a sample of world masks, in comparison to both natural faces and to painted portraits.

Whether masks are perceived and processed by the visual system in the same manner as natural faces is unclear. There is behavioral evidence that both real faces and painted representations of the face can be reliably identified as the category "face" for visual presentations as brief as $12 \mathrm{msec}$ (Graham \& Meng, 2011). Thus, artistic images appear to capture features of faces that are diagnostic in early stages of visual processing.

\footnotetext{
1 We note that researchers have recently begun to investigate perception of machine-made hyper-realistic masks, e.g., Sanders et al., 2017; Sanders and Jenkins, 2018 which seek to mimic the features of a particular face with high accuracy. This is a rather different situation compared to handmade masks from diverse global cultures.
} 
However, how higher-level aspects of visual processing respond to artistic representations of the face requires further inquiry. Therefore, Experiment 2 investigates whether the visual system engages higher-level, face-specific processing for masks using psychophysics. We use a rapid symmetry detection task involving upright and inverted masks to study this question. If masks are processed like natural faces, we should find an inversion effect: symmetry detection would be higher for upright masks than for inverted masks, since processing of upright faces presumably draws upon upright face-specific systems in cortex, whereas processing of inverted faces does not (Rhodes, Peters, Lee, Morrone, \& Burr, 2005). Given evidence of comparable low-level processing of artistic representations of the face, discussed above, we predicted that such a difference would exist.

Together, these experiments provide a first foray into the typical structure of masks and human cortical processing of masks.

\section{Experiment 1}

\subsection{Materials and Methods}

\subsubsection{Stimuli}

A photograph collection of 150 masks was used to select the initial stimuli. See Table 1 for a list of mask origins. These masks were collected from the British Museum and Metropolitan Museum of Art databases using the keyword mask. The requirements for selection were uniform lighting and frontal view. Implied gender, cultural origins, and object length and width measurements were recorded from metadata in the databases. Original images varied in size but all were at least 200 pixels in width. From this selection, 138 masks were measured and recorded as part of the experiment. The masks that were not measured were excluded due to unclear feature boundaries. Adobe Photoshop was used to record measurements using the Ruler tool.

\subsubsection{Procedure}

Masks were organized according to implied gender. There were 22 female (F), 45 male (M) and 71 unspecified (U) masks, based on image metadata. Each mask was measured in terms of pixels for the length and width of eye, mouth, \& nose features and for overall length and width (given size metadata in $\mathrm{cm}$ ). We calculated ratios of these values, with the length ratio defined as eye-mouth distance (from feature centroids) divided by mask length. Width-related information (e.g., width ratio, defined as eye separation divided by mask width) was tested in preliminary calculations but no further analysis was conducted; following earlier work on face sculpture (Di Dio, Macaluso, \& Rizzolatti, 2007) length-related data were the primary focus. See figure 3, "mask" for length and width ratio calculations and examples of measurement points.

\subsubsection{Measurement}

We measured structural features based on reference points (see Fig. 2 for average feature measures and Fig. 3 for average face structures). Eye measurements were taken for width from the inner corner (endocanthion) to outer corner (exocanthion) of each eye. Eye length measurements were taken from the upper eyelid (palpebrale superioris) to lower 
eyelid (palpebrale inferioris) of each eye. Closed or squinting eyes were measured from the crease of each eyelid (the area where the eyelid would be if the eye were open). Measurements for the two eyes were averaged together to give eye length data. Mouth width measurements were taken from the left corner (left chelion) to right corner (right chelion) of the mouth, and length was recorded from the top of the upper lip (labiale superioris) to the bottom of the lower lip (labiale inferioris). Nose length measurements were taken from the bridge of the nose to the base of the nose, and nose width measurements were taken from the outer edge of the left nostril to the outer edge of the right nostril (Costa \& Corazza, 2006).

\subsection{Results}

The average mask was 25.13 centimeters in length and 19.46 centimeters in width, which is substantially larger than the physical dimensions of the typical face (see, e.g., Du et al., 2008). Masks varied greatly in physical size as well (length $S D=22.4$, width $S D=16.82$ ). Mean structural data are given in Table 2.

Next we considered length ratio (LR). Gender generally plays a minor role in the representations of faces in masks since most representations are based on tradition, culture, or message, rather than gendered characteristics (M. Coldiron, personal communication, July 22, 2016). While the male and unspecified groups are consistent in mean LR (0.41), the female length ratio is smaller (0.39) with the least variance of the three groups $(\mathrm{SD}=0.05)$. The unspecified masks have the greatest variance in length ratio $(\mathrm{SD}=0.07)$ as well as consistently larger variation across all measurements. Female masks have the greatest mask length on average $(28.67 \mathrm{~cm})$ but in other measurements, female masks have the smallest values out of the three groups.

Length ratios of masks were then compared to length ratio data of faces and portraits from Graham et al. (2014); see Figure 4. Briefly, Graham et al. (2014) used the same measurement methods employed in the current study, which were applied to a sample of 51 Western frontal female portraits spanning 500 years of art history, and a sample of 40 frontal female faces from a standard face database. Two-sample t-tests were performed comparing data from all masks as well as data from individual mask groups ( $\mathrm{M}, \mathrm{F}$, and $\mathrm{U})$ to the sample of female portraits (mean $\mathrm{LR}=0.371$ ) and the sample of female faces (mean $\mathrm{LR}=0.357$ ). All two-sample tests comparing masks to non-masks (i.e., faces and portraits) were significant at $\mathrm{p}<0.001$ or less. We also highlight the fact that the variation in both length and width ratios of masks (length ratio $\mathrm{SD}=0.070$; width ratio $\mathrm{SD}=0.090$ ) is more than three times higher for masks than for portraits (length ratio $\mathrm{SD}=0.022$; width ratio $\mathrm{SD}=0.029$ ) or natural faces (length ratio $\mathrm{SD}=0.017$; width ratio $\mathrm{SD}=0.022$ ). Group differences in length ratio are shown in Figure 4.

We also performed a non-exhaustive examination of correlations between length ratio measurements and feature sizes within mask groups. For this analysis we removed outliers, defined as values more than 2 standard deviations away from the mean mask length (though we note that the main results of this analysis were not appreciably different with these outliers included). The final groups analyzed for correlations between features had $\mathrm{N}$ values as follows ( $\mathrm{F}: \mathrm{N}=22 ; \mathrm{M}: \mathrm{N}=43 ; \mathrm{U}: \mathrm{N}=69$ ). We found significant positive correlations $(\mathrm{p}<0.05)$ between mask length ratio and eye length for all masks 
$\left(R^{2}=0.077\right)$ and for each mask group separately ( $\left.F: R^{2}=0.18 ; M: R^{2}=0.089, U: R^{2}=0.059\right)$. We also found significant $(\mathrm{p}<0.05)$ correlations between length ratio and nose length in male $\left(R^{2}=0.14\right)$ and unspecified masks $\left(R^{2}=0.26\right)$. Mouth length was significantly correlated with length ratio for the $U$ group $\left(\mathrm{R}^{2}=0.080\right)$. Other correlations among feature variables showed lower proportions of variance and/or statistical insignificance. Overall, correlational analysis gives hints of common representational strategies among mask makers of different cultures, but clearly there is a diversity of approaches to representing the face in masks, with no clear "rule" for feature exaggeration.

\section{Experiment 2}

Perceptual anthropomorphism is defined asthe attribution of human characteristics to non-human things. This phenomenon is referred to as pareidolia in the case of face-likeness. For instance, pariedolia can occur when looking at the front of a car, a faucet, or an airplane, and emotional expression can even be evoked by such non-face objects (Desmet, Hekkert, \& Jacobs, 2000; Windhager et al., 2008). Research suggests that pareidolia may occur using neurological mechanisms for perceiving faces, such as the Fusiform Face Area (Kühn, Brick, Müller, \& Gallinat, 2014).

However, if human characteristics can be detected in objects not necessarily intended to be face-like, then it is salient to investigate the extent to which masks are processed in similar ways to natural faces. With a procedure similar to Rhodes et al. (2005), a symmetry detection task comparing upright and inverted masks was used to understand higher-level face processing of masks. Rhodes et al. (2005) found that face symmetry detection (which could be related to other high-level processing, e.g., recognition of face-ness, gender, age, etc.) is more superior for upright faces than for inverted faces. In other words, humans' powerful face-specific processing systems largely require faces to be viewed in their natural orientation, i.e., upright-despite the fact that the same features and statistical information (e.g., V1 simple cell ensemble responses) is present after inversion. To the extent that upright masks elicit a similar advantage in symmetry detection, this may indicate that high-level face-specific mechanisms are involved in the perception of masks. Thus, our procedure, stimulus creation, and participant demographics were as similar as possible to those used in Rhodes et al. (2005).

\subsection{Materials and Methods}

\subsubsection{Procedure}

In this experiment, stimuli were presented using Matlab and PsychToolBox (Brainard, 1997). Participants were shown asymmetrical and symmetrical pairs of 24 mask images in a two-interval forced choice (2IFC) task and asked to choose the symmetrical image. Each pair was shown twice, once for both possible temporal orderings (which were randomized across stimuli). The same was done for inverted versions of the same images, giving a total of 96 trials, which were presented in random order in the same session. Randomization was performed independently for each participant. Each stimulus was presented for 900 milliseconds (ms) and a 1/f-noise masking image (to eliminate retinal afterimages) was also presented for $50 \mathrm{~ms}$ after presentation of both of the two stimuli. The participant was then 
prompted with a screen asking whether the first or the second mask was symmetrical, to which they responded with a key press.

\subsubsection{Stimuli}

Although the masks from the collection in Experiment 1 have similar patterns on both sides of the face, asymmetry can be found in all masks in our collection of images. While symmetry is viewed as attractive for faces, masks are not generally intended to be symmetrical, and asymmetry can be viewed as valuable in some mask-making traditions (Edson, 2009). As masks are typically handmade, asymmetry can also be caused by imperfection in the crafting of a mask.

For example, masks created in areas such as Africa and the Northwest Coast of America are mostly made of organic materials found in the surrounding environment, which permit less precise manufacture than more processed raw materials (Mack, 1994). Furthermore, some masks may have been thrown away at the end of a ceremony or discovered long after their use, leaving them with signs of wear (Mack, 1994). Therefore, perceptible asymmetries exist in nearly all masks (as they do in nearly all faces), due to intentional and unintentional factors. Therefore, we tested a subset of 24 masks $(9 \mathrm{~F}, 10 \mathrm{M}, 5 \mathrm{U})$ from the collection used in Experiment 1 that span a range of cultures and degrees of asymmetries.

Adobe Photoshop was used to crop and blur masks and in part to create symmetrical stimuli. To diminish blemishes and isolate asymmetries in face structural features, a Gaussian blur of 1 pixel was applied to each image. All masks were reduced to a width of 200 pixels, with identical dimensions of images within each mask pair. Masks were cropped in an oval shape to exclude details other than the face.

To create the symmetrical stimuli, each mask was cut down the center, copied and flipped onto the other side of the mask to create an entirely symmetrical version-see example in Figure 5. This task was completed twice per mask, resulting in left-faced and right-faced symmetrical masks, with minor smudging edits to smooth the axis of symmetry. Using Fantamorph software, these two symmetrical versions of each mask were then morphed together at $50 \%$ each to create a symmetrical image representative of both halves of each mask.

FantaMorph was also used to create asymmetrical stimuli, morphing the original and symmetrical masks at $50 \%$. With the focus on structural asymmetry, the purpose of a morphed stimulus was to maintain an asymmetrical mask while reducing minor imperfections that could be used as cues for asymmetry besides facial structure, such as lighting differences and blemishes. The hairline of the symmetrical stimuli was maintained on the asymmetrical stimuli. The hairline was defined as the area of the mask above the eyebrows. This area of each symmetrical mask was selected, copied, and pasted onto the same area of each corresponding asymmetrical stimulus. The hairline was then blurred another 0.5 pixels on both symmetrical and asymmetrical stimuli to focus attention to the face area.

\subsubsection{Participants}


Undergraduate participants ( $\mathrm{N}=40,22$ female, 18 male) provided informed consent in accordance with the Declaration of Helsinki and received small items as incentives (thumb drive, candy, or toy sunglasses). All had normal or corrected-to-normal vision.

\subsection{Results}

The mean accuracy in symmetry detection was above chance $(50 \%)$ for both upright $(59 \%$, $\mathrm{SD}=.090)$ and inverted $(55.9 \%, \mathrm{SD}=.107)$ stimuli. One-sample t-tests comparing performance on upright and inverted faces to chance were both significant (both $\mathrm{p}<0.002$ ) - see Figure 6 . However, differences in accuracy between upright and inverted stimuli were not significant ( $\mathrm{t}$-value=1.776, $\mathrm{df}=39$ ). In contrast to the significant difference in mean performance for upright versus inverted faces reported by Rhodes et al. (2005) with a similar number of participants $(\mathrm{N}=48)$, our result can be seen as evidence that symmetry detection of upright masks may not be aided by higher-level face-specific mechanisms. However, a one-tailed t-test comparing the distribution of differences in accuracy for upright vs. inverted masks to the mean value for differences in accuracy for upright vs. inverted faces (4.4\%) reported in Rhodes et al. (2005) was not significant. On the other hand, we note that, using a two-tailed t-test, we found that the distributions of the magnitude of performance differences between upright and inverted masks (i.e., comparing performance for those who performed better for upright faces, $\mathrm{N}=23$, vs. those who performed better for inverted faces, $\mathrm{N}=15$, in terms of magnitude of performance difference) was not significant ( $\mathrm{N}=2$ performed equivalently), $\mathrm{p}=0.24$.

There was no evidence that our results were biased by a few masks that were originally very symmetrical or asymmetrical. From over 3840 trials total from 40 subjects (each pair being viewed twice), there was a mean of 34.06 errors per mask, which were relatively evenly distributed across the 24 masks ( $S D=5.13$, Median=35, Min=20, Max=45).

\section{Discussion}

\subsection{Findings}

We have provided evidence that a diverse collection of images of masks sampled from world mask-making traditions show commonalities that may be characteristic for this medium, though we emphasize that masks are an extremely diverse art form. We found that:

1. Masks in general, as well as masks separated by implied gender, differ in terms of length ratios compared to samples of female portrait paintings and of female faces. When comparing length ratio data for masks to that of human portraits, the length ratio values for masks are larger, potentially indicating further vertical exaggeration of the natural face. However, masks also show much larger variance in length ratios across our sample compared to portraits (and to natural faces), suggesting a greater diversity of representational strategies in mask-making than in portraiture. Due to the more analogous samples of faces and portraits portraying white females, perhaps the most relevant comparison would be between female faces, portraits, and masks. However, we further emphasize the diversity in mask making tradition and intention that leads to a more disparate comparison than that shown in Graham et al. (2014). 
2. Collective and gender-specific analyses for masks all showed significant correlations with eye length (although the proportion of variance explained by all investigated correlations were low). Two groups-male and unspecified-also had significant correlations with nose length. Unlike portraits, masks tend to be abstract representations: they may be more likely a projection of an idea or a symbol, rather than a realistic copy of a person's face. However, research regarding other media suggests basic inaccuracies in drawing faces: for example, untrained artists will draw features in the center of the head, despite the fact that human facial features are mostly in the bottom half of the head (Ostrofsky, Kozbelt, Tumminia, \& Cipriano, 2016); similar effects could be at work in the 3-D medium of masks, and may warrant investigation.

3. Despite these commonalities and empirical patterns in mask structure-as well as the fact that masks are often worn over the face-we found evidence consistent with the notion that high-level face processing mechanisms were not recruited during perception of masks. This raises questions about human mask making and justifies the need for further investigation. For example, if masks are not processed in the same way as faces, can humans reliably detect emotional expressions in them, as has been implied by past research? Are masks deliberately designed to deviate from real faces, perhaps as a way of avoiding the "uncanny valley" separating reality and replication of humanity (Bartneck, Kanda, Ishiguro, \& Hagita, 2007)? And what is the role of wearing a mask over the face during performance in terms of perception and processing of emotion? That is, does natural positioning on the body and motion with the wearer influence perceptual processing of masks? These questions also demand further investigation.

From a visual neuroscience point of view, our work raises additional questions: In what ways does brain processing of masks differ from that of faces? Some have argued that the "specialness" of human face perception may be exaggerated, at least with regard to unfamiliar faces. However, even unfamiliar faces show a host of specialized effects and processing in the brain (Young \& Burton, 2017). Moreover, both humans and monkeys have been shown to exhibit cognitive and behavioral responses to natural face-like images, and other primates have been shown to experience pareidolia (Taubert, Wardle, Flessert, Leopold, \& Ungerleider, 2017). Thus, even when a viewer can be sure that a given object is not a face, it seems that the brain is especially reactive to its face-likeness. Masks offer an interesting avenue for addressing these questions since mask makers and viewers have been performing natural experiments in face perception in diverse cultures for millennia.

\subsection{Limitations}

This preliminary study undoubtedly comes with some limitations. Although our mask compilation was as diverse as possible, the influence of particular cultures and regions on mask structure was not a variable considered in the analysis. Our sample is thus a biased one. Further research might consider cultural and historical elements of masks, mask-makers, and other artistic face representations to gain a deeper understanding of how faces are represented and perceived in artistic renderings. Furthermore, our analyses compared all masks with painted portraits, without considering the difference in intention behind each artistic face representation. Painted portraits are typically focused on realism, whereas not all masks in our sample were intended to be realistic, but are rather a 
representation of an idea or value (Mack, 1994). To think more critically about each art form, as well as face representation as a whole, one might consider medium and technology as factors. For instance, the tools used by a portrait painter may result in a very different representation than a mask maker using stone, bone or wood with a different set of tools, even if both artists are modeling the same face. To accommodate these additional components of art, masks and portraits, as well as face representation, future research could perform within-sample analysis to learn more about varied face representations within masks alone, as well as compare masks with other forms of face representation, such as sculpture and computerized faces. Further studies could also examine biases inherent in the creation process for 3-D representations of the face.

\section{Conclusions}

We have made an initial foray into the study of human perception of masks. Our work shows that human mask makers have a wide variety of representational strategies, which are reflected in the basic structure of the represented face. Given this diversity of representations - which is augmented by myriad variations in decoration that we did not quantify - we asked whether masks demonstrate an inversion effect, such that perception of the mask is disrupted by inversion. We found that performance on a symmetry detection task was not significantly different for upright versus inverted masks, which is consistent with the idea that humans' powerful, high-level face processing systems may not be invoked when viewing masks.

Why, then are masks produced in so many cultures, and so often worn over the face? Further investigation is needed to determine the threshold for object similarity to a natural face. However, as noted previously by art historians (Edson, 2009; Mack, 1994), we conclude that masks may generally serve purposes that do not rely on naturalistic representation of the face, despite masks' superficial functionality as face surrogates.

Acknowledgments: We thank Margaret Coldiron (University of Essex) for her many helpful comments during this project. We received support for this work from a HWS Faculty Research Grant to D.G.

Conflicts of Interest: The authors declare no conflicts of interest.

Data Availability: A file containing mask image origins and data is available at: http://people.hws.edu/graham/maskdata.zip

\section{References}

Bartneck, C., Kanda, T., Ishiguro, H., \& Hagita, N. (2007). Is the Uncanny Valley an Uncanny Cliff? Paper presented at the Proceedings of the 16th IEEE International Symposium on Robot and Human Interactive Communication, RO-MAN 2007.

Bednarik, R. (2006). The Lower and Middle Palaeolithic Origins of Semiotics 3, 89-107.

Brainard, D. H. (1997). The Psychophysics Toolbox. Spat Vis, 10(4), 433-436.

Bruce, V., \& Young, A. (2012). Face perception. New York, NY, US: Psychology Press.

Calder, A., Rhodes, G., Johnson, M., \& Haxby, J. (2011). Oxford Handbook of Face Perception: Oxford University Press. 
Costa, M., \& Corazza, L. (2006). Aesthetic phenomena as supernormal stimuli: the case of eye, lip, and lower-face size and roundness in artistic portraits. Perception, 35(2), 229-246. doi:10.1068/p3449

Desmet, P., Hekkert, P., \& J Jacobs, J. (2000). When a car makes you smile: Development and application of an instrument to measure product emotions (Vol. 27).

Di Dio, C., Macaluso, E., \& Rizzolatti, G. (2007). The Golden Beauty: Brain Response to Classical and Renaissance Sculptures. PLOS ONE, 2(11), e1201. doi:10.1371/journal.pone.0001201

Du, L. L., Wang, L. M., \& Zhuang, Z. (2008). Measurement and analysis of human head-face dimensions. Zhonghua lao dong wei sheng zhi ye bing za zhi= Zhonghua laodong weisheng zhiyebing zazhi= Chinese journal of industrial hygiene and occupational diseases, 26(5), 266-270.

Edson, G. (2009). Masks and masking: Faces of tradition and belief worldwide: McFarland.

Germine, L., Russell, R., Bronstad, P. M., Blokland, G. A., Smoller, J. W., Kwok, H., . . . Wilmer, J. B. (2015). Individual Aesthetic Preferences for Faces Are Shaped Mostly by Environments, Not Genes. Curr Biol, 25(20), 2684-2689. doi:10.1016/j.cub.2015.08.048

Graham, D., \& Meng, M. (2011). Altered spatial frequency content in paintings by artists with schizophrenia. i-Perception, 2(1), 1-9.

Graham, D., Pallett, P. M., Meng, M., \& Leder, H. (2014). Representation and Aesthetics of the Human Face in Portraiture. Art \& Perception, 2(1-2), 75-98. doi:https://doi.org/10.1163/22134913-00002026

Kühn, S., Brick, T. R., Müller, B. C. N., \& Gallinat, J. (2014). Is This Car Looking at You? How Anthropomorphism Predicts Fusiform Face Area Activation when Seeing Cars. PLOS ONE, 9(12), e113885. doi:10.1371/journal.pone.0113885

Mack, J. (1994). Masks: the art of expression: British Museum Press.

Ostrofsky, J., Kozbelt, A., Tumminia, M., \& Cipriano, M. (2016). Why do non-artists draw the eyes too far up the head? How vertical eye-drawing errors relate to schematic knowledge, pseudoneglect, and context-based perceptual biases. Psychology of Aesthetics, Creativity, and the Arts, 10(3), 332.

Rhodes, G., Peters, M., Lee, K., Morrone, M. C., \& Burr, D. (2005). Higher-level mechanisms detect facial symmetry. Proceedings of the Royal Society of London B: Biological Sciences, 272(1570), 1379-1384.

Sanders, J. G., \& Jenkins, R. (2018). Individual differences in hyper-realistic mask detection. Cognitive research: principles and implications, 3(1), 24.

Sanders, J. G., Ueda, Y., Minemoto, K., Noyes, E., Yoshikawa, S., \& Jenkins, R. (2017). Hyper-realistic face masks: a new challenge in person identification. Cognitive research: principles and implications, 2(1), 43.

Taubert, J., Wardle, S. G., Flessert, M., Leopold, D. A., \& Ungerleider, L. G. (2017). Face pareidolia in the rhesus monkey. Current Biology, 27(16), 2505-2509. e2502.

Windhager, S., Slice, D. E., Schaefer, K., Oberzaucher, E., Thorstensen, T., \& Grammer, K. (2008). Face to face. Human Nature, 19(4), 331-346. 
Young-Laughlin, J., \& Laughlin, C. D. (1988). How Masks Work, or Masks Work How? Journal of Ritual Studies, 59-86.

Young, A. W., \& Burton, A. M. (2017). Are We Face Experts? Trends Cogn Sci. 


\begin{tabular}{|c|c|c|c|}
\hline $\begin{array}{l}\text { Region \& } \\
\text { Subculture(s) }\end{array}$ & $\mathrm{n}$ & \begin{tabular}{|l|} 
Region \& \\
Subculture(s)
\end{tabular} & $\mathrm{n}$ \\
\hline Alaska & 4 & Italy & 8 \\
\hline Eskimo & 1 & Campania & 1 \\
\hline Tlingit & 1 & Rome & 1 \\
\hline Yup'ik & 1 & Ivory coast & 8 \\
\hline Angola & 1 & Baule & 1 \\
\hline Bangladesh & 1 & Dan & 4 \\
\hline Belize & 2 & Guro & 2 \\
\hline Mopan & 2 & Senufo & 1 \\
\hline Borneo & 1 & Japan & 14 \\
\hline Burma & 1 & Edo Period & 3 \\
\hline Cameroon & 5 & Heian Period & 1 \\
\hline Bamum & 1 & Netsuke & 4 \\
\hline Banyang & 1 & Korea & 1 \\
\hline Bum & 1 & Yi Dynasty & 1 \\
\hline Fungom & 1 & Liberia & 2 \\
\hline Widekum & 1 & Bassa & 1 \\
\hline Canada & 10 & Dan & 1 \\
\hline Haida & 7 & Malawi & 3 \\
\hline Inuit & 1 & Malaysia & 2 \\
\hline Kwakwaka'wakw & 1 & Berawan & 1 \\
\hline Salish & 1 & Iban & 1 \\
\hline China & 3 & Mali & 1 \\
\hline Liao Dynasty & 1 & Dogon & 1 \\
\hline Ordos & 1 & Mexico & 9 \\
\hline Xinjiang & 1 & Olmec & 1 \\
\hline Zhou Dynasty & 1 & Teotihuacan & 4 \\
\hline Colombia & 4 & Tlatilco & 1 \\
\hline Arhuaco & 1 & Mongolia & 1 \\
\hline Tolita-Tumaco & 3 & Nepal & 5 \\
\hline Cyprus & 2 & Kathmandu & 2 \\
\hline Late Hellenistic & 1 & Malla & 1 \\
\hline Dem. Rep. of the & 8 & New Caledonia & 1 \\
\hline Congo & & Kanak & 1 \\
\hline Luba & 1 & Papua New Guinea & 8 \\
\hline Lega & 1 & Buna & 1 \\
\hline Lwalu & 1 & Kapriman & 1 \\
\hline Pende & 1 & Krosmeri & 1 \\
\hline Suku & 1 & Tolai & 2 \\
\hline Zimba & 1 & Umboi or Siassi & 1 \\
\hline East Timor & 1 & Wogumas & 1 \\
\hline Egypt & 4 & Peru & 8 \\
\hline Ptolemaic & 1 & Cashinahua & 1 \\
\hline Roman Period & 2 & Moche & 7 \\
\hline France & 2 & Queensland & 1 \\
\hline Gabon & 3 & Torres Strait & 1 \\
\hline Fang & 1 & Islander & \\
\hline Punu & 2 & Romania & 2 \\
\hline Greece & 6 & Russia & 1 \\
\hline Cypriot & 1 & Sahka & 1 \\
\hline Greenland & 1 & South Arabia & 1 \\
\hline Inuit & 1 & Sabaean & 1 \\
\hline Guatemala & 3 & South Korea & 1 \\
\hline Maya & 1 & Buddhist & 1 \\
\hline India & 11 & Sri Lanka & 4 \\
\hline Kashmir & 1 & Buddhist & 1 \\
\hline Monba & 3 & Tibet & 3 \\
\hline West Bengal & 1 & \begin{tabular}{|l|} 
Turkey \\
\end{tabular} & 1 \\
\hline Indonesia & 20 & Roman & 1 \\
\hline Bali & 7 & United States & 2 \\
\hline Central Javanese & 1 & Cherokee & 1 \\
\hline Karo Batak & 1 & Lenape & 1 \\
\hline Toba Batak & 1 & Vanuatu & 1 \\
\hline Java & 7 & Ambrym Island & 1 \\
\hline Iraq & 2 & Zambia & 1 \\
\hline Kassite & 1 & & \\
\hline
\end{tabular}

Table 1

Table 1. Origins of masks used in this study, categorized by region and subculture as labeled in original databases (British Museum, Metropolitan Museum of Art). Quantities within each category and subcategory are listed. 


\begin{tabular}{|lllll|}
\hline Mean Value & All Masks & Female & Male & Unspecified \\
\hline Length Ratio [SD] & $0.41[.07]$ & $0.39[.05]$ & $0.41[.06]$ & $0.41[.07]$ \\
Width Ratio [SD] & $0.49[.09]$ & $0.52[.08]$ & $0.47[.08]$ & $0.50[.11]$ \\
Mask Length $(\mathrm{cm})[\mathrm{SD}]$ & $25.13[22.40]$ & $28.67[17.01]$ & $23.50[17.73]$ & $25.07[26.31]$ \\
Mask Width $(\mathrm{cm})[\mathrm{SD}]$ & $19.46[16.82]$ & $18.53[9.42]$ & $20.03[17.35]$ & $19.39[18.25]$ \\
Eye Length $(\mathrm{cm})[\mathrm{SD}]$ & $2.21[2.29]$ & $1.93[1.37]$ & $1.97[2.16]$ & $2.46[2.57]$ \\
Nose Length $(\mathrm{cm})[\mathrm{SD}]$ & $6.50[8.19]$ & $5.42[2.63]$ & $6.04[4.74]$ & $7.13[10.69]$ \\
Mouth Length $(\mathrm{cm})[\mathrm{SD}]$ & $2.80[2.96]$ & $2.44[1.97]$ & $2.68[2.94]$ & $2.99[3.25]$ \\
\hline
\end{tabular}

Table 2. Measures of length ratio, width ratio, and measurements of mask length, width, and nose, eye, and mouth measurements $(\mathrm{cm})$ in terms of average values and standard deviation (in parentheses). These data are for all masks, as well as groups: female, male, and unspecified. 


\section{Figures}
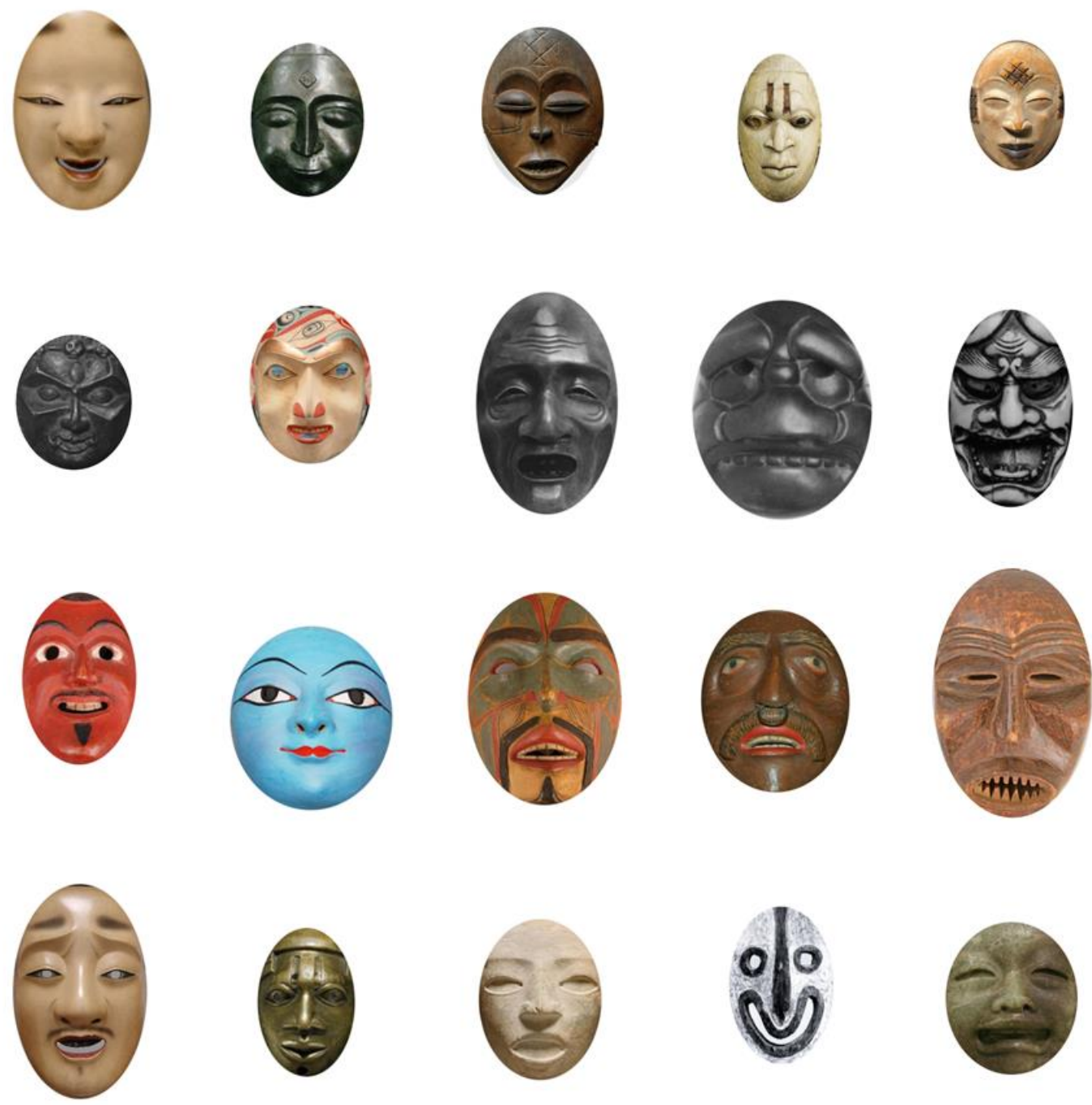

Figure 1. Examples of masks from different cultures, cropped to isolate the face. This set is a subset of the masks used in Experiment 2, themselves a subset of the 138 masks used in Experiment 1. 


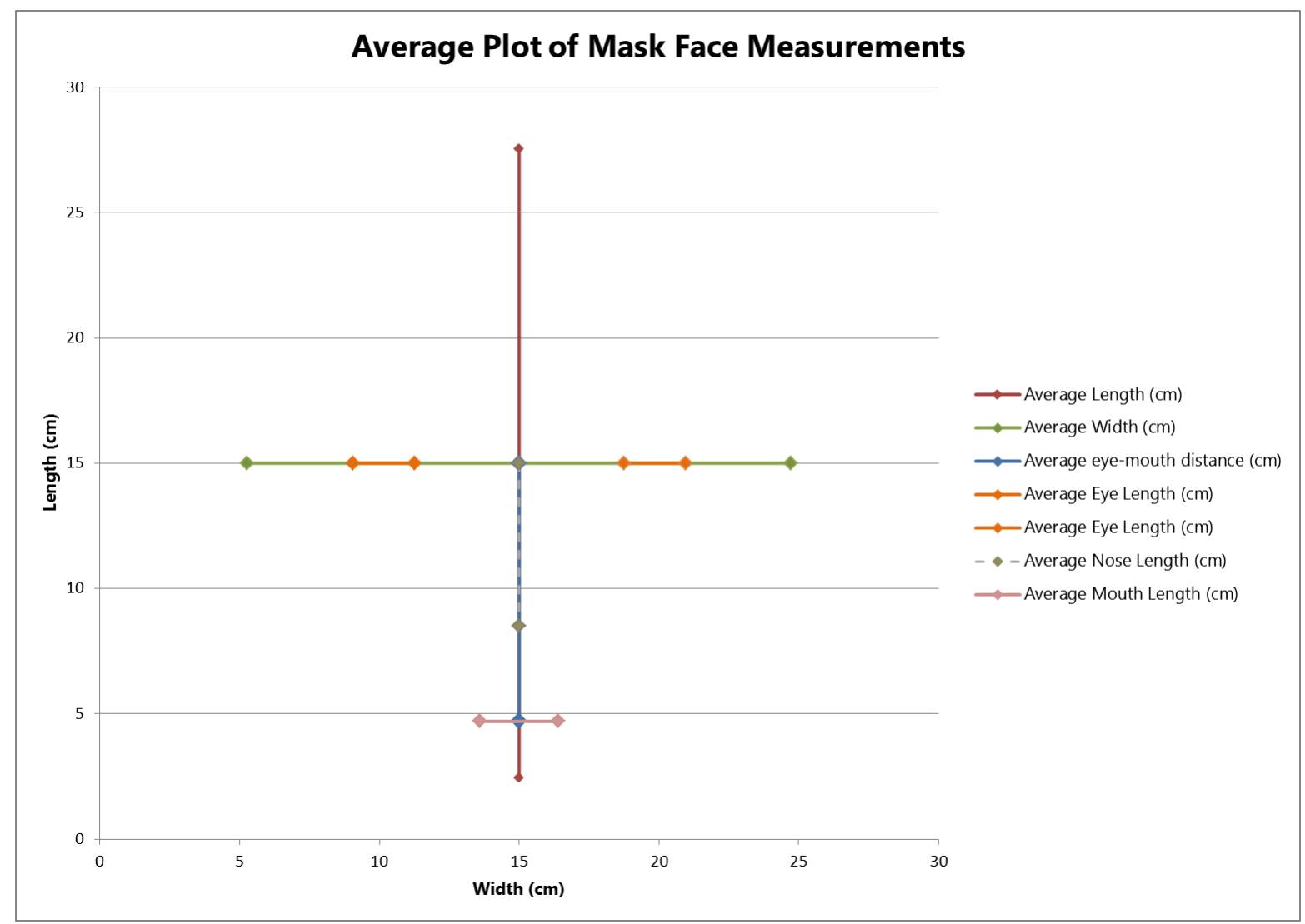

Figure 2. Average mask facial feature measures, on a $30 \mathrm{~cm}-$ by $-30 \mathrm{~cm}$ coordinate plot to demonstrate average locations of features. 


\section{Mask}

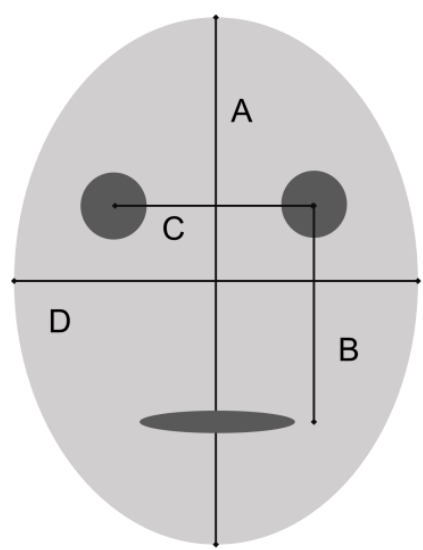

Length Ratio $(B / A)=0.41$

Width Ratio $(C / D)=0.49$
Portrait

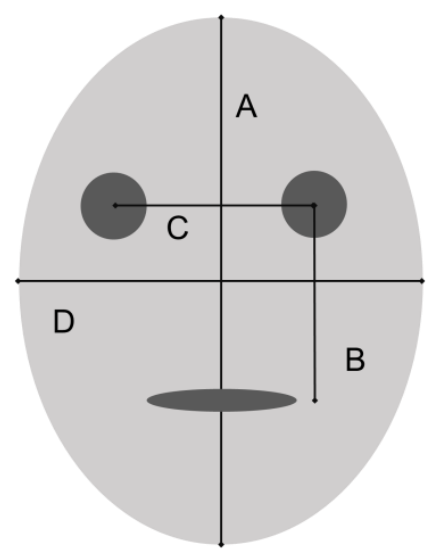

Length Ratio $(B / A)=0.37$

Width Ratio $(C / D)=0.49$

\section{Face}

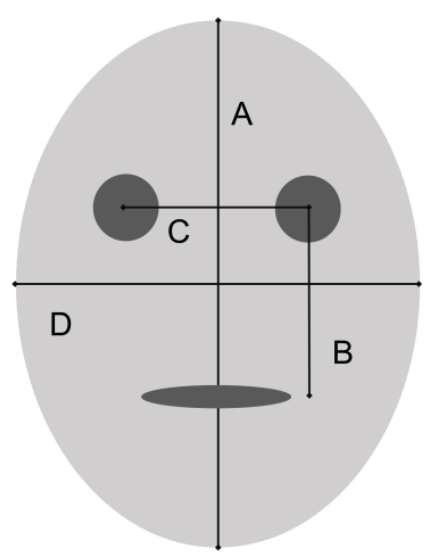

Length Ratio $(B / A)=0.36$

Width Ratio $(C / D)=0.46$

Figure 3. Comparison of average face structure of masks as compared to painted portraits and natural faces (portrait and face data from Graham et al., 2014), including measurement points for face length (A), eye-mouth distance (B), eye distance (C), face width (D) and computation of length and width ratios. 


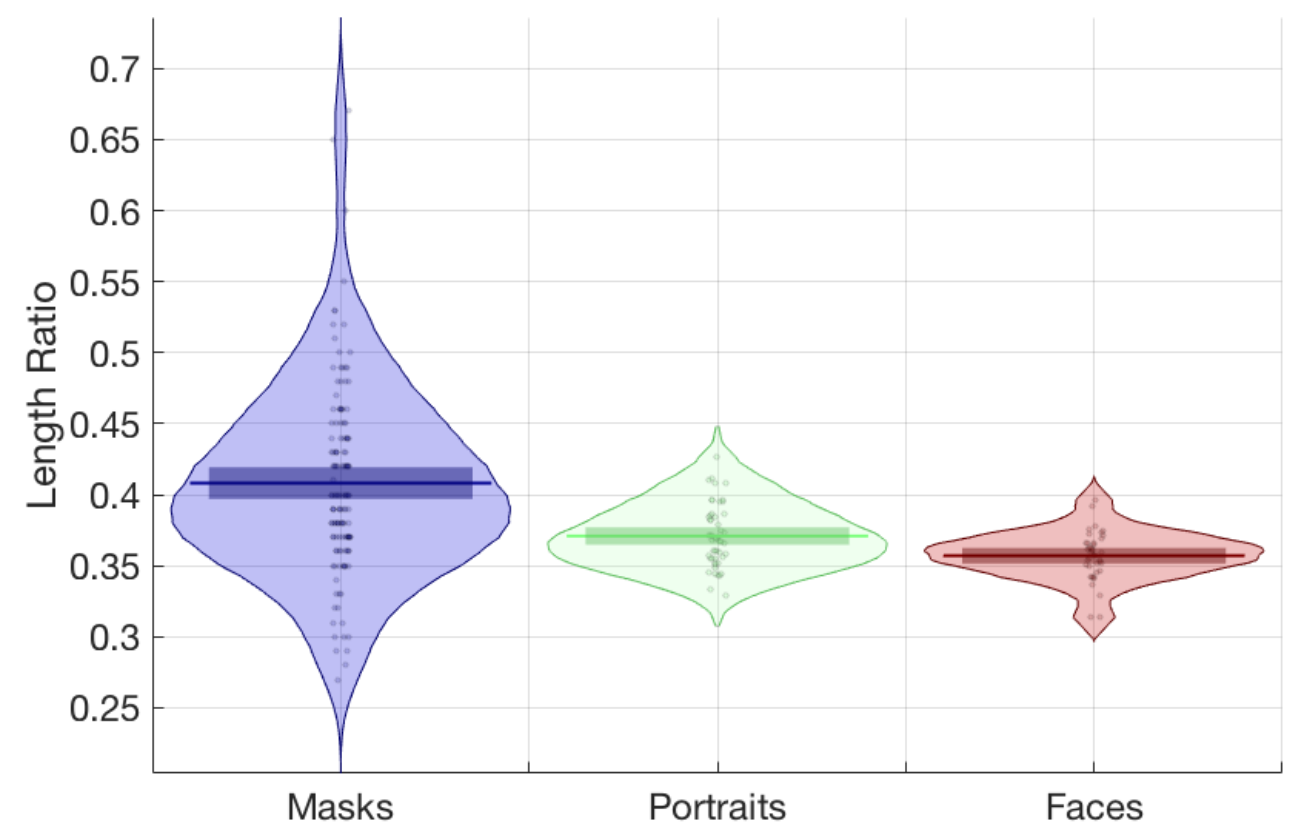

Figure 4. Average length ratio measurements (dark horizontal bar) of masks as compared to painted portraits and natural faces (portrait and face data from Graham et al., 2014), including histograms of underlying data in the vertical direction. Masks were significantly different from both portraits and faces at the level of $\mathrm{p}<0.001$. 


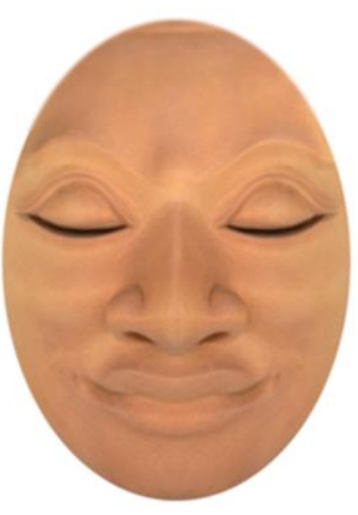

(a)

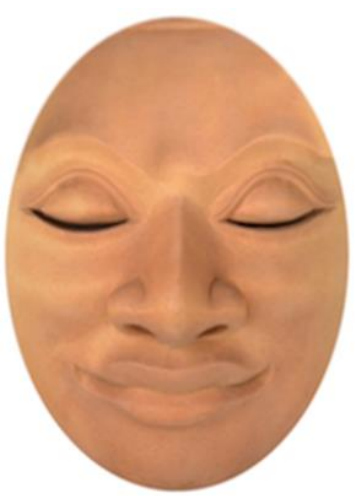

(b)

Figure 5. An example (U64) of symmetrical (a) and asymmetrical (b) stimuli used in Experiment 2. Jauk Mask; Bali, Indonesia; 1870s-1931; Wood; British Museum Accession: As1932,0406.32. 


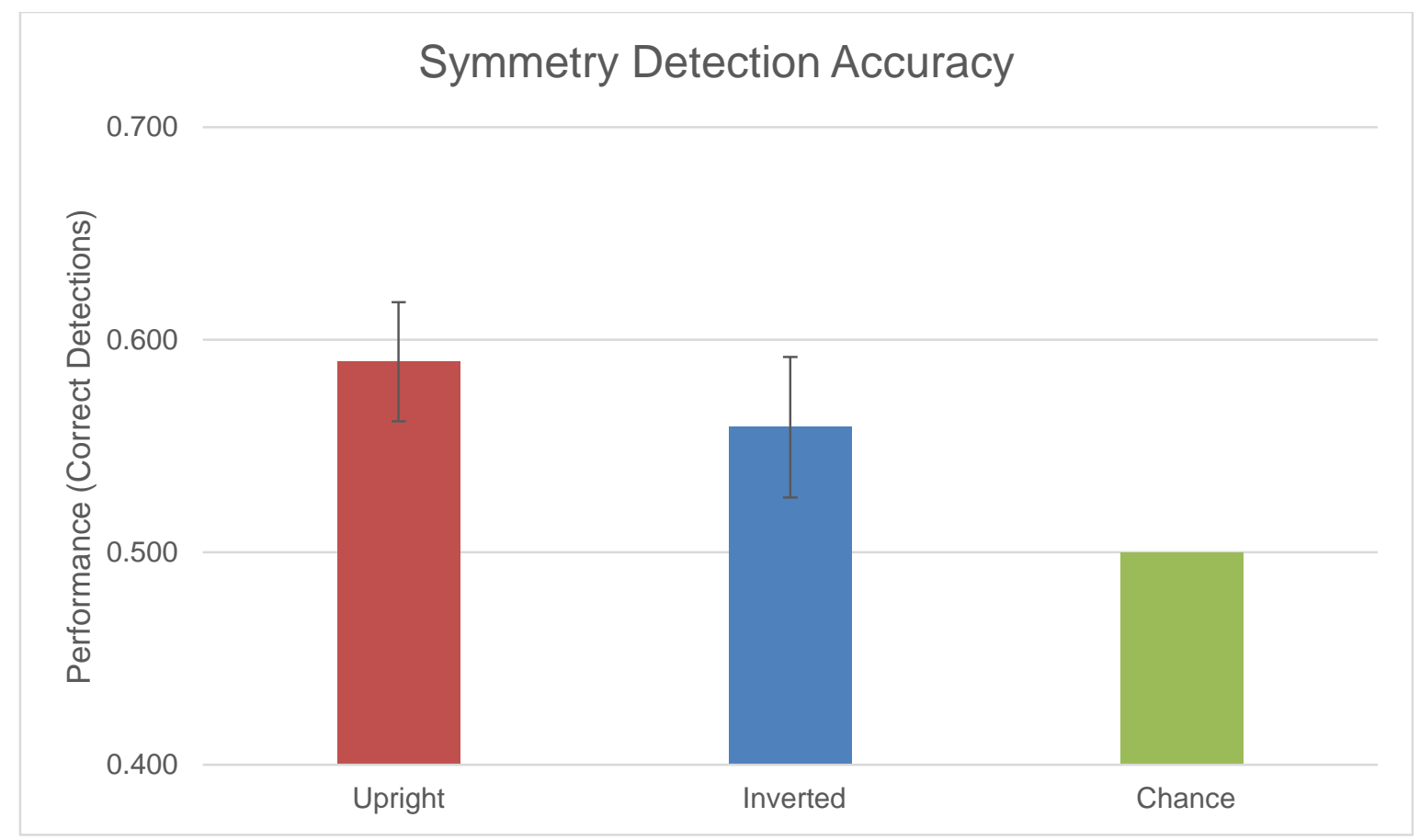

Figure 6. Results of Experiment 2. This experiment investigated mean percentages of correctly identified symmetrical masks in a 2IFC paradigm, for both upright and inverted masks. Error bars indicate 95\% confidence intervals. Upright and inverted faces are not significantly different $(p>0.05)$ from one another but both are significantly different $(\mathrm{p}<0.002)$ from chance $(50 \%)$. 